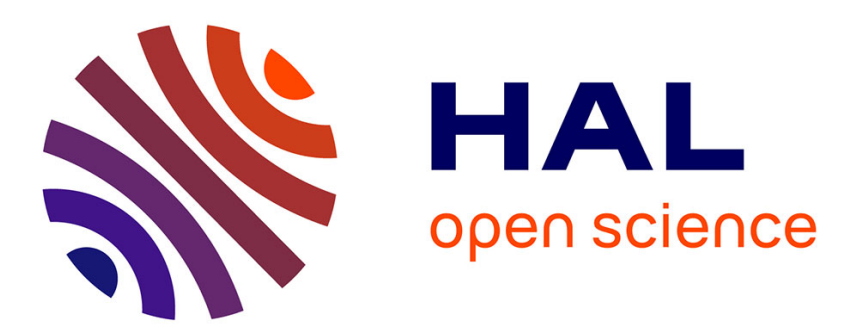

\title{
Steady and Unsteady Buckling of Viscous Capillary Jets and Liquid Bridges
}

Jingxuan Tian, Neil M. Ribe, Xiaoxiao Wu, Ho Cheung Shum

\section{To cite this version:}

Jingxuan Tian, Neil M. Ribe, Xiaoxiao Wu, Ho Cheung Shum. Steady and Unsteady Buckling of Viscous Capillary Jets and Liquid Bridges. Physical Review Letters, 2020, 125 (10), 10.1103/PhysRevLett.125.104502 . hal-02932114

\section{HAL Id: hal-02932114 https://hal.science/hal-02932114}

Submitted on 7 Sep 2020

HAL is a multi-disciplinary open access archive for the deposit and dissemination of scientific research documents, whether they are published or not. The documents may come from teaching and research institutions in France or abroad, or from public or private research centers.
L'archive ouverte pluridisciplinaire HAL, est destinée au dépôt et à la diffusion de documents scientifiques de niveau recherche, publiés ou non, émanant des établissements d'enseignement et de recherche français ou étrangers, des laboratoires publics ou privés. 


\title{
Steady and Unsteady Buckling of Viscous Capillary Jets and Liquid Bridges
}

\author{
Jingxuan Tian ${ }^{1, a)}$, Neil M. Ribe ${ }^{2, a), b)}$, Xiaoxiao $\mathrm{Wu}^{3}$, and Ho Cheung Shum ${ }^{1, c)}$ \\ ${ }^{1}$ Department of Mechanical Engineering, The University of Hong Kong, Pokfulam Road, Hong Kong, China \\ ${ }^{2}$ Lab FAST, University Paris-Saclay, CNRS, Bât. 530, Campus Univ, 91400 Orsay, France and \\ ${ }^{3}$ Faculties of Sciences and Engineering, The University of Hong Kong, Pokfulam Road, Hong Kong, China*
}

(Dated: July 25, 2020)

\begin{abstract}
Steady buckling (coiling) of thin falling liquid jets is sensitive to surface tension, yet an understanding of these capillary effects lags far behind what is known about surface tension-free coiling. In experiments with sub-millimetric jets and ultra-low flow rates, we find that the critical dispensing height $H_{c}$ for coiling decreases with increasing flow rate, a trend opposite to that found previously for inertia-free coiling. We resolve the apparent contradiction using nonlinear numerical simulations based on slender-jet theory, which show that the trend reversal is due to the strong effect of surface tension in our experiments. We use our experiments to construct a regime diagram (coiling vs. stagnation flow) in the space of capillary number $C a$ and jet slenderness $\epsilon$, and find that it agrees well with fully nonlinear numerical simulations. However, it differs substantially from the analogous regime diagram determined experimentally by Le Merrer et al. (2012) for the unsteady buckling of a compressed liquid bridge. Using linear stability analysis, we show that the differences between the two regime diagrams can be explained by a combination of shape nonuniformity and the influence of gravity.
\end{abstract}

An elastic rod compressed along its axis buckles when the load exceeds a critical value, a phenomenon first studied by Euler in the 18th century. Liquid 'rods' can also buckle under compression; here the resisting force is due to viscosity. A liquid bridge compressed sufficiently rapidly between rigid pistons eventually bends; this is an example of unsteady buckling [19]. By contrast, steady buckling occurs when the liquid falls continuously downward from a nozzle onto a stationary surface. This 'liquid rope coiling' (LRC), has been intensively studied during the past 60 years using laboratory experiments, linear stability analysis, asymptotic analysis and numerical simulation $[2-5,11,17,18,21,23,25,26]$. LRC is interesting not only for the rich dynamics emerging from the interplay of the multiple forces involved, but also because it is encountered in practical situations such as additive manufacturing of functional materials $[13,15,16,20,28]$ and ultra-fine viscous liquid dispensing $[6-8,14,27]$. The high resolution involved here is achieved by dispensing very thin jets at very low flow rates. The effects of surface tension should therefore be particularly strong. Yet all existing studies of LRC consider vanishing [21, 25] or weak $[12,18]$ surface tension. Understanding the coiling of capillary jets with strong surface tension is essential not only for completing the big picture of LRC dynamics, but also for a variety of applications in high-resolution extrusion-based printing and liquid dispensing.

Here we combine experiments, nonlinear numerical simulation and linear stability analysis (LSA) to reveal the dynamics of surface tension-dominated LRC in the limit of negligible inertia. We gain further understanding by comparing steady buckling with unsteady buckling [19] using LSA. To our knowledge, ours is the first study to elucidate LRC for capillary jets, using three independent approaches that agree closely with one another.

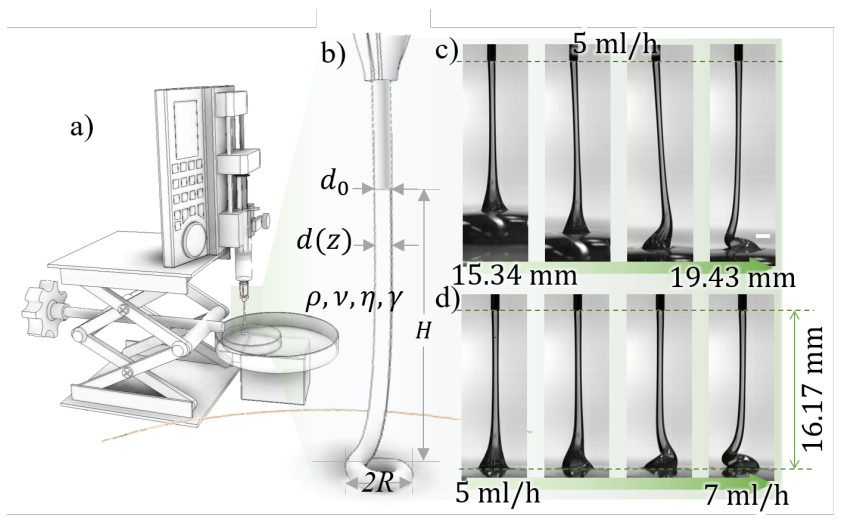

FIG. 1: (a) Experimental setup. (b) Schematic of the extruded jet with density $\rho$, kinematic viscosity $\nu$, dynamic viscosity $\eta$, surface tension coefficient $\gamma$, nozzle diameter $d_{0}$ and jet diameter $d(z)$. (c) Photographs of the jet for fixed flow rate $Q$ and increasing fall height $H$, showing the transition from stagnation flow to coiling. The scale bar is $1 \mathrm{~mm}$. (d) Still photographs of a similar transition at a fixed fall height with increasing flow rate.

Experimental procedure. We used silicone oils (Sigma Aldrich) with viscosities $3.2-60 \mathrm{~Pa}$ s measured by a rheometer (Anton Paar). The fluid was extruded from a glass syringe activated by a syringe pump fixed to an elevatable platform (fig. 1a). Fig. 1b shows a schematic of the jet. The flow rate ranged from $1.5 \mathrm{~mL} / \mathrm{h}$ to 150 $\mathrm{mL} / \mathrm{h}$. Visual data was recorded by high-speed camera (Photon).

Critical height versus flow rate. As the fall height increases beyond a critical value $H_{c}$ with the flow rate held fixed, an initially straight jet exhibits incipient buckling that quickly turns into finite-amplitude coiling (fig. 1c). A similar transition occurs as the flow rate increases for a fixed dispensing height (fig. 1d). Fig. 2 shows $H_{c}$ 


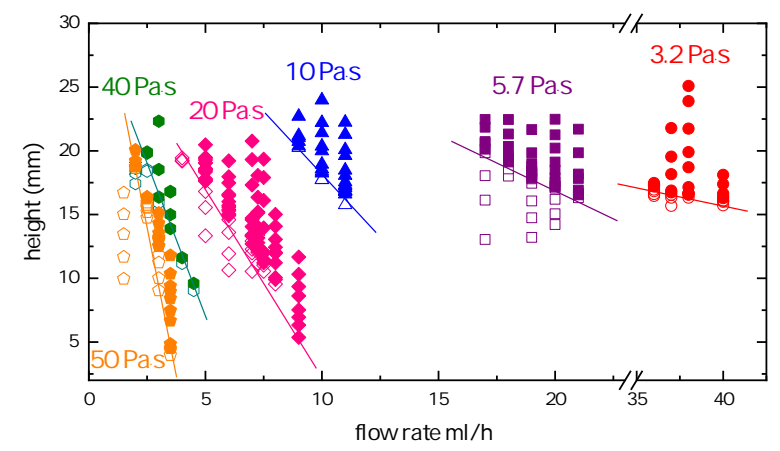

FIG. 2: Experimentally determined critical height for the onset of coiling as a function of flow rate and viscosity. The needle diameter is $d_{0}=0.6 \mathrm{~mm}$. Different colors correspond to different viscosities as indicated. Solid symbols represent coiling, whereas open symbols correspond to stagnation flow. Solid lines are drawn by eye to fit the boundaries between open and solid symbols to indicate a decreasing trend.

(solid lines) as a function of flow rate $Q$ for 6 sets of experiments with different viscosities. $H_{c}$ decreases with $Q$ within each set. This is opposite to the trend previously found $[12,24]$ for surface tension-free coiling with negligible inertia, for which $H_{c}$ is either independent of flow rate ('viscous' regime) or increases with flow rate ('gravitational' regime).

One clue to the cause of this discrepancy is the scaleddown character of our experiments. Compared to previous studies $[2,4,10,12]$, we use nozzle diameters $\left(d_{0}=0.6\right.$ $1.55 \mathrm{~mm})$ smaller by a factor of $\approx 2$ and flow rates $(Q$ $=1.5-150 \mathrm{~mL} / \mathrm{h})$ smaller by $\approx 2$ orders of magnitude. A comparison of parameters is found in [1] table S1. Another clue is that our critical heights $H_{c}$ are as large as $\approx 93 d_{0}$, about 15 times the largest values $\approx 5-6 d_{0}$ from the existing literature ([1] table S2). Both clues suggest the hypothesis that the discrepancy is due to strong surface tension. To test this, we use a slender-jet model [21] implemented in the continuation and bifurcation software AUTO-07P [9] to simulate coiling with strong surface tension. In the limit of negligible inertia, the critical height $H_{c}$ can depend only on $d_{0}, Q, \nu$, $\rho g$, and $\gamma$. The $\Pi$-theorem of dimensional analysis then implies that three independent dimensionless groups can be formed from these six parameters. We choose these groups to be

$$
\frac{H_{c}}{d_{0}}, \quad\left(\frac{\nu Q}{g d_{0}^{4}}\right)^{1 / 4} \equiv \Pi_{Q}, \quad \frac{\rho g d_{0}^{2}}{\gamma} \equiv B o
$$

where $\Pi_{Q}$ is a dimensionless flow rate and the Bond number $B o$ is the ratio of gravitational to capillary forces. Following [22], we define $H_{c}$ as the height at which the numerically predicted radius $a_{1}$ of the lowermost part of the coiling jet just equals the radius $R$ of the coil itself, because $a_{1}>R$ corresponds to unphysical self-penetration. Fig. 3 shows our calculated critical heights (solid lines)

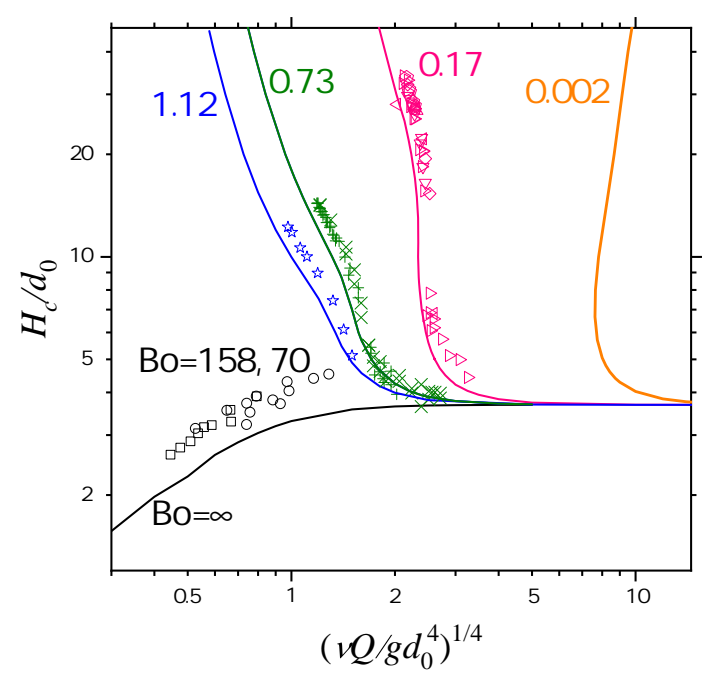

FIG. 3: Numerically predicted (lines) and experimentally measured (symbols) critical heights $H_{c}$ vs. $\Pi_{Q}$ for several values of the Bond number $B o([1]$ note $\mathrm{S} 4)$. The black line replicates a previous result for $B o=\infty[22]$ and is compared with experimental data of [4] for $B o=158$ (squares) and 70 (circles). The other four curves are for the values of $B o$ indicated. The symbols represent different viscosities: 3.2 $\mathrm{Pa} \mathrm{s}$ (up-pointing triangles), 5.7 $\mathrm{Pa} \mathrm{s}$ (pentagons), $10 \mathrm{~Pa} \mathrm{~s}$ (right-pointing triangles, stars and + symbol), $20 \mathrm{~Pa}$ s (downpointing triangles), $30 \mathrm{~Pa} \mathrm{~s}$ (x symbol), $40 \mathrm{~Pa} \mathrm{~s}$ (diamonds), and $50 \mathrm{~Pa} \mathrm{~s}$ (left-pointing triangles). The corresponding onset frequencies (both measured and predicted) are shown in [1] fig. S1.

together with our experimental measurements (symbols) as functions of $\Pi_{Q}$ for several values of $B o$. Despite a small systematic offset, the measured data points and the calculated curves track each other well. For large $B o \geq 70$ (negligible surface tension), $H_{c}$ increases with the flow rate, whereas it decreases when $B o \leq 1.12$ (strong surface tension). This is the apparent contradiction that we pointed out earlier between the results of this study and those of $[4,5,12]$; we now see that it is due to the large effect of surface tension in our experiments.

Regime diagram for buckling. Steady buckling (LRC) is induced by injecting fluid into the upper end of a jet while keeping the fall height fixed. By contrast, in unsteady buckling the height of a compressed liquid bridge decreases while the deflection of the centerline increases monotonically. Yet while steady and unsteady buckling show these obvious differences, a deeper physical understanding of both can be obtained by comparing them. To do this we construct a regime diagram (LRC vs. stagnation flow) in the space of jet slenderness $d_{0} / H \equiv \epsilon$ and capillary number $\eta U_{0} / \gamma \equiv C a$, following the procedure used by [19] for unsteady buckling. Here $U_{0}=4 Q / \pi d_{0}^{2}$ is the mean extrusion velocity, and $C a=(4 / \pi) \Pi_{Q}^{4} B o$. Fig. 4 shows our experimental data for steady buckling in the $\epsilon-C a$ plane, together with the critical curve (black dashed line) for unsteady buckling of [19]. One sees immediately 


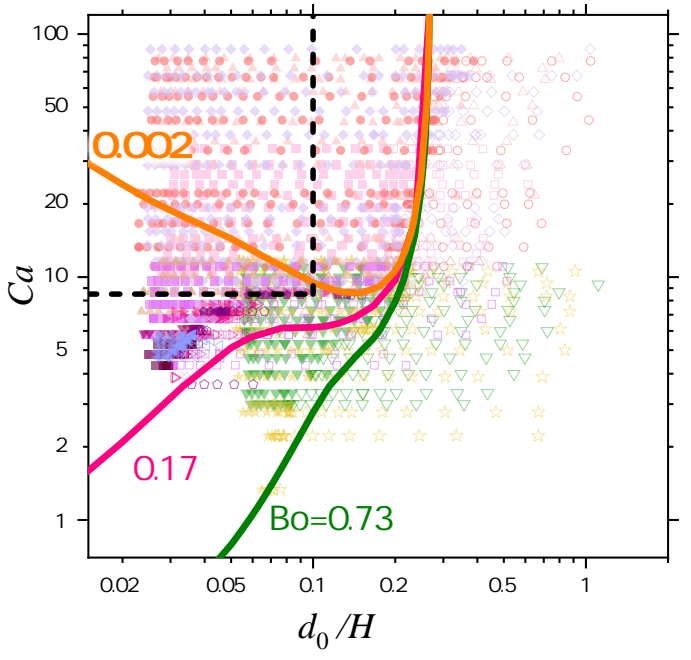

FIG. 4: Regime diagram for steady buckling (LRC) in the space of jet slenderness $d_{0} / H \equiv \epsilon$ and capillary number $\eta U_{0} / \gamma \equiv C a$. Solid lines indicate the stagnation/coiling transition predicted by nonlinear numerical simulations of LRC for $B o=0.73,0.17$ and 0.002 . The dashed black line is the critical curve for unsteady buckling [19]. Experimental data are indicated by filled symbols (coiling) and open symbols (stagnation flow). The pink/violet symbols are for $B o=0.17$, and the yellow/green symbols are for $B o=0.73$. Viscosities used are 3.2 $\mathrm{Pa} \mathrm{s}$ (deep-plum downward-pointing triangles), 5.7 Pa s (orchid left-pointing triangles), $10 \mathrm{~Pa}$ s (light-pink squares), 20 $\mathrm{Pa}$ s (deep-pink right-pointing triangles), $23 \mathrm{~Pa}$ s (blush-pink circles), $30 \mathrm{~Pa}$ s (light-brown triangles), $40 \mathrm{~Pa} \mathrm{~s}$ (light-violet diamonds), and $50 \mathrm{~Pa} \mathrm{~s}$ (purple pentagons) for $B o=0.17$; $10 \mathrm{~Pa} \mathrm{~s}$ (yellow stars) and $30 \mathrm{~Pa} \mathrm{~s}$ (green downward-pointing triangles) for $B o=0.73$. Analogous results for $B o=0.032$, which like the case $B o=0.002$ exhibits multiple onset heights, can be found in [1] note S5 and the supplemental video.

that the domain of steady buckling (filled symbols) is larger than that of unsteady buckling, extending both to lower values of $C a<8.5$ and to higher values of $\epsilon>0.1$.

Also shown in fig. 4 are critical curves for steady buckling calculated numerically for three values of $B o$. $B o=0.73$ corresponds to the experimental data shown in green/yellow, while $B o=0.17$ corresponds to pink/violet data. The good agreement of the calculated critical curves with the experimentally observed regime boundaries shows that the minimum value of $C a$ for which buckling occurs depends strongly on $B o$, i.e. on the effect of gravity relative to surface tension. Gravity is relatively unimportant in the upper part of the diagram where the three critical curves coincide.

Linear stability analysis. We now turn to LSA, which unlike the nonlinear slender-jet model can be applied to both steady (jet) and unsteady (bridge) buckling, allowing us to compare them consistently. The analysis will elucidate the distinct causes of the greater rightward (to larger $\epsilon$ ) and downward (to smaller $\mathrm{Ca}$ ) extents of the steady buckling region relative to the unsteady buckling region in fig. 4. We first demonstrate the relevance of LSA by using it to predict accurately the critical buckling height and frequency for one of our experiments on LRC ([1] Note S1). Now we apply LSA to unsteady buckling of a compressed liquid bridge.

Without loss of generality we suppose that buckling is confined to the $x_{1}-x_{3}$ plane, where $x_{3} \equiv z$ increases downward. We further suppose that the lateral deflection $\zeta$ of the jet's axis is small. In the absence of inertia, the equations of force balance perpendicular to and parallel to the jet's axis are [23]

$$
\begin{gathered}
-M_{2}^{\prime \prime}+\kappa_{2} N_{3}+\frac{\pi}{2} \gamma d \kappa_{2}-\rho A g \zeta^{\prime}=0 \\
N_{3}^{\prime}+\frac{\pi}{2} \gamma d^{\prime}+\rho A g=0,
\end{gathered}
$$

where $N_{3}(z)$ is the normal viscous force on the crosssection, $M_{2}(z, t)$ is the bending moment, $A=\pi d^{2} / 4$, $\kappa_{2} \equiv \zeta^{\prime \prime}(z, t)$ is the curvature of the axis about the $x_{2}$-direction and primes denote $\partial / \partial z$. The constitutive relations for $N_{3}$ and $M_{2}$ are $N_{3}=3 \eta A U_{3}^{\prime}$ and $M_{2}=3 \eta I\left(\dot{\zeta}^{\prime \prime}+U_{3} \zeta^{\prime \prime \prime}-U_{3}^{\prime} \zeta^{\prime \prime} / 2\right)[26]$, where $I=\pi d^{4} / 64$, $U_{3}$ is the vertical velocity on the jet's axis, and a dot indicates $\partial / \partial t$.

We now combine the foregoing equations and set $\zeta=$ $F(z) \exp \sigma t$, where $\sigma$ is the growth rate. The resulting equations for $U_{3}$ and $F$ are

$$
\begin{gathered}
3 \eta\left(d^{2} U_{3}^{\prime}\right)^{\prime}+2 \gamma d^{\prime}+\rho g d^{2}=0 \\
{\left[d^{4}\left(\sigma F^{\prime \prime}+U_{3} F^{\prime \prime \prime}-\frac{1}{2} U_{3}^{\prime} F^{\prime \prime}\right)\right]^{\prime \prime}} \\
-16\left(d^{2} U_{3}^{\prime}+\frac{2 \gamma}{3 \eta} d\right) F^{\prime \prime}+\frac{16 \rho g}{3 \eta} d^{2} F^{\prime}=0 .
\end{gathered}
$$

Equation (3b) together with the 'clamped' boundary conditions $F(0)=F^{\prime}(0)=F^{\prime \prime}(0)=F(H)=F^{\prime}(H)=0$ is an eigenvalue problem for $F(z)$ that can be solved if $d(z)$ and $U_{3}(z)$ are known. A critical difference between a clamped liquid bridge and a flowing jet is that the diameter $d(z)$ is highly nonuniform in the former case. To approximate the shape of a static liquid bridge before it is compressed, we assume $d=d_{0}+\left(d_{1}-d_{0}\right)(2 z-H)^{2} / H^{2}$, where $d_{0}$ is the diameter in the center and $d_{1}$ the diameter at either end. Fig. 5a shows this shape for $d_{1} / d_{0}=2.2$, a value determined by least-squares fitting of the assumed quadratic shape to the central $50 \%$ of the bridge shown in the leftmost image of fig. 1a of [19]. The known function $d(z)$ is now injected into (3a) with $g=0$, which can then be solved analytically subject to $U_{3}(0)=U_{0}$ and $U_{3}(H)=0$ ([1] Note S2.) The eigenvalue problem for $F(z)$ is then solved using AUTO-07p. The only eigen- 
a)

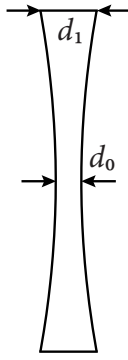

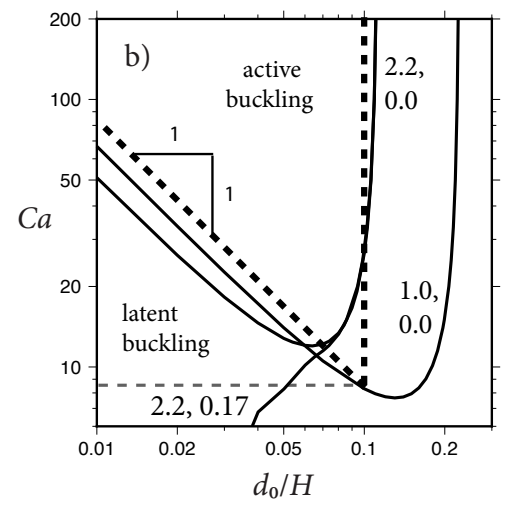

FIG. 5: Results of linear stability analysis of unsteady basic states of a compressed liquid bridge. (a) Prescribed parabolic shape of the bridge for $d_{1} / d_{0}=2.2$. (b) Critical curves for unsteady buckling in the $d_{0} / H-C a$ space. The three solid curves are predicted by linear stability analysis for the values of $\left(d_{1} / d_{0}, B o\right)$ indicated. The dashed lines bound the regions of active and latent unsteady buckling of [19].

values are two positive real ones, which lie on a doublevalued (folded) surface above the $\epsilon-C a$ plane. These correspond to amplified buckling without oscillation, as observed by [19]. To determine where in the $\epsilon-C a$ plane such growing modes exist, we start from either mode and continue it to higher $\epsilon$ and/or lower $C a$ until a turning point is encountered beyond which the solution no longer exists. The locus of all such turning points is a critical curve that divides the $\epsilon$ - $C a$ plane into two regions where buckling occurs and does not occur.

Fig. 5b shows those critical curves (solid lines) for three combinations of values of $d_{1} / d_{0}$ and $B o$. The dashed lines bound the regions of active and latent unsteady buckling proposed by [19] ([1] Note S3), such that bridges initially in the latent region remain straight while their slenderness $\epsilon$ increases until they reach the active region. The 'reference' critical curve for $\left(d_{1} / d_{0}, B o\right)=$ $(2.2,0.0)$ agrees well with the boundaries of the active buckling region of [19]. Relative to this, the curve with $\left(d_{1} / d_{0}, B o\right)=(1.0,0.0)$ shows that buckling of a uniform bridge occurs for values of the slenderness $\epsilon$ that are about twice as great as those for a nonuniform bridge with $d_{1} / d_{0}=2.2$. This is because the thicker ends of the nonuniform bridge resist buckling more effectively than the thinner ends of the uniform bridge. In summary, the maximum slenderness $d_{0} / H$ for which unsteady buckling occurs depends strongly on the shape of the bridge.

Continuing with fig. 5b, we explore the effect of gravity by comparing the reference curve with the curve for $d_{1} / d_{0}=2.2$ and $B o=0.17$, the values in the experiments of [19]. Whereas the two curves coincide for $\mathrm{Ca}>12$, they are quite different for lower values of $\mathrm{Ca}$, and even the signs of their slopes are opposite. Active unsteady buckling should occur everywhere above and to the left of the curve with $B o=0.17$. However, this is not what was seen in the experiments of [19], who observed buckling only above a horizontal line $C a=8.5$. The reason for this disagreement between experiment and theory is unclear.

Discussion. This work began as an effort to understand why the critical height for steady buckling of capillary jets decreases as a function of flow rate, a trend opposite to that seen in previous studies of inertia-free coiling. The small nozzle sizes and flow rates used in our experiments, as well as our observations of unusually long stable jets, suggested that the trend reversal was due to strong surface tension, a hypothesis that we confirmed with fully nonlinear slender-jet numerical simulations. The importance of surface tension relative to viscous forces in steady buckling can also be estimated roughly as the characteristic ratio of the surface tension force $F_{\gamma} \sim \gamma a_{1} R^{-1}$ to the viscous force $F_{v} \sim \rho \nu a_{1}^{4} U_{0} R^{-4}$, where $R$ is the coil radius, $a_{1}$ is the radius of the jet within the coil, and $U_{1} \equiv Q / \pi a_{1}^{2}$ is the axial velocity of the fluid in the coil [21]. We find that $F_{\gamma} / F_{v}=1.1-2.3$ for our experiments, indicating the crucial importance of surface tension.

We gained further physical insight by comparing our experimental phase diagram for steady buckling in the $\epsilon-C a$ plane with an analogous diagram for unsteady buckling [19]. There are two major differences between the two diagrams. First, steady buckling occurs up to $\epsilon \approx 0.25$, greater than the maximum slenderness $\epsilon \approx 0.1$ for unsteady buckling. Second, steady buckling occurs for capillary numbers as low as 1.3, whereas unsteady buckling is limited to $C a \geq 8.5$. These two differences have distinct causes that we were able to elucidate using finite-amplitude numerical simulation and linear stability analysis. The different ranges of $\epsilon$ are explained by a difference in shape of the buckling object: a steadily coiling capillary jet has a nearly constant diameter, whereas a buckling liquid bridge has strongly thickened ends that oppose a greater resistance to bending. The cause of the different ranges of $\mathrm{Ca}$ is more subtle. First, note that all three of our independent approaches (experiments for steady buckling, numerical simulation of steady coiling, and LSA of unsteady buckling) agree in showing that the minimum value of $C a$ for buckling decreases as gravity becomes more important relative to surface tension, i.e. as $B o$ increases. This result would explain the difference between our regime diagram and that of [19] if gravity were negligible in the latter study. This is not so, however, because $B o=0.17$ in the experiments of [19]. According to our LSA, a gravitational effect of this magnitude relative to surface tension should permit unsteady buckling to occur well below the lower limit $\mathrm{Ca}=8.5$ observed by [19]. Further analysis and experiments are needed to resolve this contradiction.

Our work has important applications to micro-scale manipulation of viscous fluid threads in situations such as high-resolution printing of intricate patterns and func- 
tional architectures. The current trend towards ever smaller scales means that surface tension will play a crucial role in future applications. The present work may therefore provide timely guidance for practice. On the theoretical side, asymptotic analysis similar to that of [3] could help to elucidate the detailed mechanism by which strong surface tension reverses the trend of the variation of onset height as a function of flow rate. In closing, we note that our investigation of the effects of surface tension has been limited to coiling in the inertia-free viscous and gravitational regimes. Future research on the influence of surface tension on inertial coiling will bring us closer to a complete physical picture of liquid rope coiling.

This work was supported by the General Research Fund (Nos. 17306315 and 17329516) and the Research Impact Fund (R7072-18) of the University of Hong Kong and by the PROCORE France/Hong Kong Joint Research Scheme (Nos. 40424RF and F-HKU703/17) from the Partenariat Hubert Curien and the Research Grants Council of Hong Kong. The authors thank the anonymous referees for their helpful comments.

* a). J. Tian and N. M. Ribe contributed equally to this work.

b). Corresponding author. ribe@fast.u-psud.fr

c). Corresponding author. ashum@hku.hk

[1] See supplemental material [url].

[2] G. Barnes and R. Woodcock. Liquid rope-coil effect. Am. J. Physics, 26:205-209, 1958.

[3] M. J. Blount. Bending and Buckling of a Falling Viscous Thread. PhD thesis, University of Cambridge, 2010.

[4] J. O. Cruickshank. Viscous Fluid Buckling: A Theoretical and Experimental Analysis with Extensions to General Fluid Stability. PhD thesis, Iowa State University, 1980.

[5] J. O. Cruickshank and B. R. Munson. Viscous fluid buckling of plane and axisymmetric jets. J. Fluid Mech., 113:221-239, 1981.

[6] T. Cubaud, B. Jose, and S. Darvishi. Folded microthreads: Role of viscosity and interfacial tension. Phys. Fluids, 23:042002, 2011.

[7] T. Cubaud and T. G. Mason. Folding of viscous threads in diverging microchannels. Phys. Rev. Lett., 96:114501, 2006.

[8] S. Darvishi and T. Cubaud. Formation of capillary structures with highly viscous fluids in plane microchannels. Soft Matter, 8:10658-10668, 2012.

[9] E. J. Doedel, T. F. Fairgrieve, B. Sandstede, A. R. Champneys, Y. A. Kuznetsov, and X. Wang. Auto-07p: Continuation and bifurcation software for ordinary dif- ferential equations. Technical report, 2007.

[10] M. Habibi, S. H. Hosseini, M. H. Khatami, and N. M. Ribe. Liquid supercoiling. Phys. Fluids, 26:024101, 2014.

[11] M. Habibi, M. Maleki, R. Golestanian, N. M. Ribe, and D. Bonn. Dynamics of liquid rope coiling. Phys. Rev. E, 74:066306, 2006.

[12] M. Habibi, Y. Rahmani, D. Bonn, and N. M. Ribe. Buckling of liquid columns. Phys. Rev. Lett., 104:074301, 2010.

[13] H.-Y. Kim, M. Lee, K. J. Park, S. Kim, and L. Mahadevan. Nanopottery: Coiling of electrospun polymer nanofibers. Nano Lett., 10:2138-2140, 2010.

[14] T. Kong, J. Li, Z. Liu, Z. Zhou, P. H. Y. Ng, L. Wang, and H. C. Shum. Rapid mixing of viscous liquids by electrical coiling. Sci. Rep., 6:19606, 2016.

[15] J. Li, T. Kong, J. Yu, K. H. Lee, Y. H. Tang, K. Kwok, J. T. Kim, and H. C. Shum. Electrocoiling-guided printing of multiscale architectures at single-wavelength resolution. Lab Chip, 19:1953-1960, 2019.

[16] J. I. Lipton and H. Lipson. 3d printing variable stiffness foams using viscous thread instability. Sci. Rep., 6:29996, 2016.

[17] L. Mahadevan, W. S. Ryu, and A. D. T. Samuel. Correction: Fluid 'rope trick' investigated. Nature, 403:502, 2000 .

[18] M. Maleki, M. Habibi, R. Golestanian, N. M. Ribe, and D. Bonn. Liquid rope coiling on a solid surface. Phys. Rev. Lett., 93:214502, 2004.

[19] M. Le Merrer, D. Quéré, and C. Clanet. Buckling of viscous filaments of a fluid under compression stress. Phys. Rev. Lett., 109:064502, 2012.

[20] R. Passieux, L. Guthrie, S. H. Rad, M. Lévesque, D. Therriault, and F. P. Gosselin. Instability-assisted direct writing of microstructured fibers featuring sacrificial bonds. Adv. Mater., 27:3676-3680, 2015.

[21] N. M. Ribe. Coiling of viscous jets. Proc. R. Soc. London A, 460:3223-3239, 2004.

[22] N. M. Ribe. Liquid rope coiling: a synoptic view. $J$. Fluid Mech., 812:R2, 2017.

[23] N. M. Ribe, M. Habibi, and D. Bonn. Stability of liquid rope coiling. Phys. Fluids, 18:084102, 2006b.

[24] N. M. Ribe, M. Habibi, and D. Bonn. Liquid rope coiling. Ann. Rev. Fluid Mech., 44:249-266, 2012.

[25] N. M. Ribe, H. E. Huppert, M. A. Hallworth, M. Habibi, and D. Bonn. Multiple coexisting states of liquid rope coiling. J. Fluid Mech., 555:275-297, 2006a.

[26] B. Tchavdarov, A. L. Yarin, and S. Radev. Buckling of thin liquid jets. J. Fluid Mech., 253:593-615, 1993.

[27] J. Tian, J. Li, A. Sauret, T. Kong, X. Wu, Y. Lu, and H. C. Shum. Facile control of liquid-rope coiling with tunable electric field configuration. Phys. Rev. Applied, 12:014034, Jul 2019.

[28] H. Yuk and X. Zhao. A new 3d printing strategy by harnessing deformation, instability, and fracture of viscoelastic inks. Adv. Mater., 30:1704028, 2017. 


\title{
Steady and Unsteady Buckling of Viscous Capillary Jets and Liquid Bridges Supplemental Material
}

\author{
Jingxuan $\operatorname{Tian}^{1, a)}$, Neil M. Ribe ${ }^{2, a), b)}$, Xiaoxiao $\mathrm{Wu}^{3}$, and Ho Cheung Shum ${ }^{1, c)}$ \\ 1 Department of Mechanical Engineering, The University of Hong Kong, Pokfulam Road, Hong Kong, China \\ ${ }^{2}$ Lab FAST, University Paris-Saclay, CNRS, Bât. 530, Campus Univ, 91400 Orsay, France and \\ ${ }^{3}$ Faculties of Sciences and Engineering, The University of Hong Kong, Pokfulam Road, Hong Kong, China*
}

Figure $\mathbf{S 1 . O n s e t ~ f r e q u e n c y ~ o f ~ c o i l i n g ~ a s ~ a ~ f u n c t i o n ~ o f ~}$ dimensionless flow rate. In addition to onset height, we also calculated the onset frequency with our nonlinear slender-jet code. The experimental coiling frequency was extracted from videos using fast Fourier transformation. The agreement between experiments and numerics is less good for onset frequency than for onset height, although the trends of the experimental observations are captured by the numerics.

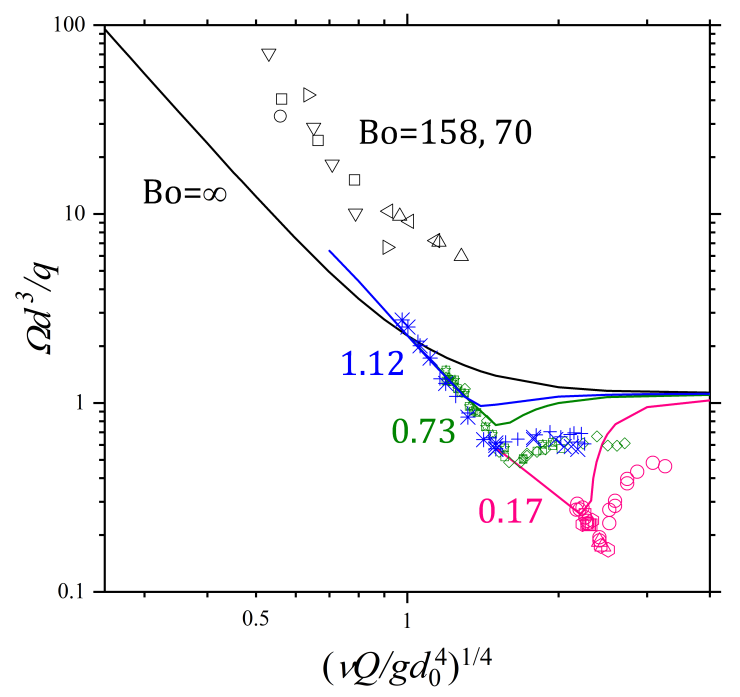

FIG. S1: Coiling onset frequency as a function of flow rate for Bond numbers $B o=\infty$ (black), 1.12 (blue), 0.73 (green) and 0.17 (pink). Solid lines: numerical predictions of the nonlinear slender-jet model. Symbols: experimental measurements. Viscosities used are 3.4 $\mathrm{Pa} \mathrm{s}$ (pink squares), 5.8 $\mathrm{Pa} \mathrm{s}$ (pink circles), $10 \mathrm{~Pa} \mathrm{~s}$ (pink triangles), $22 \mathrm{~Pa}$ s (pink hexagons), 10 $\mathrm{Pa}$ s (blue +), $30 \mathrm{~Pa} \mathrm{~s}$ (blue $\mathrm{X}$ ), $10 \mathrm{~Pa}$ s (olive squares), 30 $\mathrm{Pa}$ s (olive diamonds).

Note S1. Linear stability analysis of steady buckling To verify the relevance of linear stability analysis to the phenomenon of buckling, we first apply it to a steady basic state similar to one observed in our experiments. The equations governing the dynamics are (4a) and (4b) in the main text, which are valid for both steady and unsteady buckling.

Fig. S2a shows a photograph of the basic state we shall consider, which has the parameters indicated in the figure caption. To mimic this state numerically, we first eliminate $d(z)$ from eqn. (4a) in the main text using the steady flux conservation relation $d(z)^{2} U_{3}(z)=d_{0}^{2} U_{0}$. The resulting equation for $U_{3}(z)$ is then solved numerically subject to the boundary conditions $U_{3}(0)=U_{0}$ and $U_{3}(H)=0.1096 U_{0}$, where the constant 0.1096 was calculated from the diameter of the bottom of the jet in the photograph of fig. S2a. Finally, the diameter $d(z)$ of the jet is calculated from the steady flux conservation relation written down previously. The resulting shape is shown in the rightmost part of Fig S2a. To analyze the linear stability of this steady state, we solved equation (4b) in the main text numerically subject to the boundary conditions $F(0)=F^{\prime}(0)=F(H)=F^{\prime}(H)=$ $F^{\prime \prime}(0)=0$. The first four of these boundary conditions state that the jet's axis is effectively clamped at both ends. The fifth condition states that the curvature of the jet's axis is zero at the nozzle, which in physical terms means that material elements of fluid exiting the nozzle have a zero rate of rotation about the $x_{2}$-axis. Because the five boundary conditions are all homogeneous, equation $4 \mathrm{~b}$ defines an eigenvalue problem that has nontrivial solutions only for particular values of $\sigma$. We found the eigenvalues and associated eigenmodes using AUTO-07P, following the procedure of [8]. For the state of fig. S2a there exists a slightly subcritical mode with a complex eigenvalue $\sigma$ having a small negative real part. Continuing this mode to greater values of $H$, we found that $\operatorname{Re}(\sigma)$ vanishes at $H_{c}=18 \mathrm{~mm}$, corresponding to the onset of buckling with a frequency of $1.14 \mathrm{~Hz}$. The experimentally measured critical height and frequency are $19.8 \mathrm{~mm}$ and $1.21 \mathrm{~Hz}$, which differ from the predicted values by $9 \%$ and $6 \%$, respectively. Fig. S2b shows the real and imaginary parts of the neutrally stable mode $F(z)$. The real part is only nonzero in the lowermost part of the jet where the axial stress is compressive, as required for buckling. By contrast, the imaginary part is nonzero over the whole height range, corresponding to periodic motion of the whole jet.

Note S2. Axial velocity in a compressed liquid bridge. We consider a liquid bridge having a nonuniform diameter

$$
d(z)=d_{0}+\left(d_{1}-d_{0}\right)(2 z-H)^{2} / H^{2},
$$

where $d_{0}$ and $d_{1}>d_{0}$ are the minimum and maximum diameters at the center and the two ends, respectively. Substituting (1) into eqn. (3a) in the main text yields a second-order ordinary differential equation for $U_{3}(z)$ 

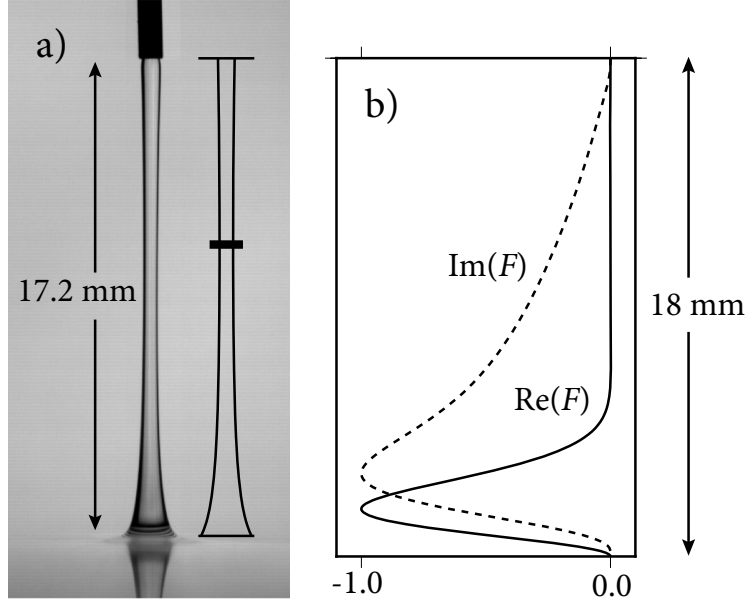

FIG. S2: Linear stability analysis of a steady basic state of a flowing jet. (a) Photograph of a jet with $d_{0}=0.6 \mathrm{~mm}$, $H=17.2 \mathrm{~mm}, \eta=10 \mathrm{~Pa} \mathrm{~s}$, and $Q=10 \mathrm{~mL} / \mathrm{h}$. Superimposed on the photograph is the jet shape calculated numerically for the same parameters and a bottom diameter $3.02 d_{0}$. The axial stress is extensional above the horizontal black line and compressional below it. (b) Real and imaginary parts of the neutrally stable mode at $H=18 \mathrm{~mm}$, normalized to their maximum absolute values.

that can be solved analytically subject to the boundary conditions $U_{3}(0)=U_{0}$ and $U_{3}(H)=0$. The solution is

$$
\begin{gathered}
D \frac{U_{3}}{U_{0}}=\phi \hat{d}_{1} \tan ^{-1} \phi\left(\phi ^ { 2 } \left(45 \hat{d}_{1} \epsilon^{2}\left(d_{1}(1-2 s)^{2}-4(s-1) s\right)\right.\right. \\
+C a^{-1}\left(-8 B o(s-1) s-3 B o \hat{d}_{1}^{2}(1-2 s)^{2} s(s-1)\right. \\
\left.\left.+6 \hat{d}_{1}\left(2 B o(s-1)^{2} s^{2}+5 \epsilon(2 s-1)\right)\right)\right) \\
+B o C a^{-1} \hat{d}_{1}\left(\hat{d}_{1}(1-2 s)^{2}\right. \\
\left.-4(s-1) s)\left(\ln \hat{d}_{1}-\ln \left(\hat{d}_{1}(1-2 s)^{2}-4(s-1) s\right)\right)\right) \\
-\left(\phi ^ { 2 } \left(\phi ^ { 2 } ( s - 1 ) \left(8 B o C a^{-1} s-3 \hat{d}_{1}\left(\hat{d}_{1}(2 s-1)\right.\right.\right.\right. \\
\left.\left.\left(30 \epsilon^{2}+B o C a^{-1}(1-2 s) s\right)+4 s\left(-15 \epsilon^{2}+B o C a^{-1}(s-1) s\right)\right)\right) \\
+\hat{d}_{1}\left(\hat{d}_{1}(1-2 s)^{2}-4(s-1) s\right)\left(15 \phi \epsilon\left(2 C a^{-1}+3 \hat{d}_{1} \epsilon\right)\right. \\
\tan ^{-1}(\phi(2 s-1))+B o C a^{-1} \\
\left.\left.\left.\left(-\ln \hat{d}_{1}+\ln \left(\hat{d}_{1}(1-2 s)^{2}-4(s-1) s\right)\right)\right)\right)\right)
\end{gathered}
$$

where $\epsilon=d_{0} / H, \hat{d}_{1}=d_{1} / d_{0}, s=z / H, \phi=\sqrt{\hat{d}_{1}-1}$, and

$$
D=90 \epsilon^{2} \hat{d}_{1} \phi^{3}\left(\hat{d}_{1}(1-2 s)^{2}-4(s-1) s\right)\left(\phi+\hat{d}_{1} \tan ^{-1} \phi\right),
$$

Note S3. Active vs. latent unsteady buckling. Strictly speaking, buckling only occurs in the wedgeshaped region between the heavy dashed lines $\epsilon=0.1$ and $C a=0.85 \epsilon^{-1}$ in Fig. 5 in the main text. We shall call this the "active" buckling region. In the laboratory, however, [7] also observed buckling for points $(\epsilon, \mathrm{Ca})$ located in the wedge between the light dashed line $(C a=8.5)$ and the lower of the two heavy dashed lines $\left(C a=0.85 \epsilon^{-1}\right)$. We call this the "latent" buckling region. The reason why buckling is observed in this region is that the experiments of [7] were performed at a constant speed of compression, that is, at a constant capillary number $C a$. As it is compressed, a liquid bridge initially in the latent buckling region will see its slenderness $\epsilon$ continually increase while $C a$ remains constant. This corresponds to a rightward movement in the $(\epsilon, C a)$ plane. Buckling then begins when the liquid bridge reaches the left boundary $C a=0.85 \epsilon^{-1}$ of the active buckling region.

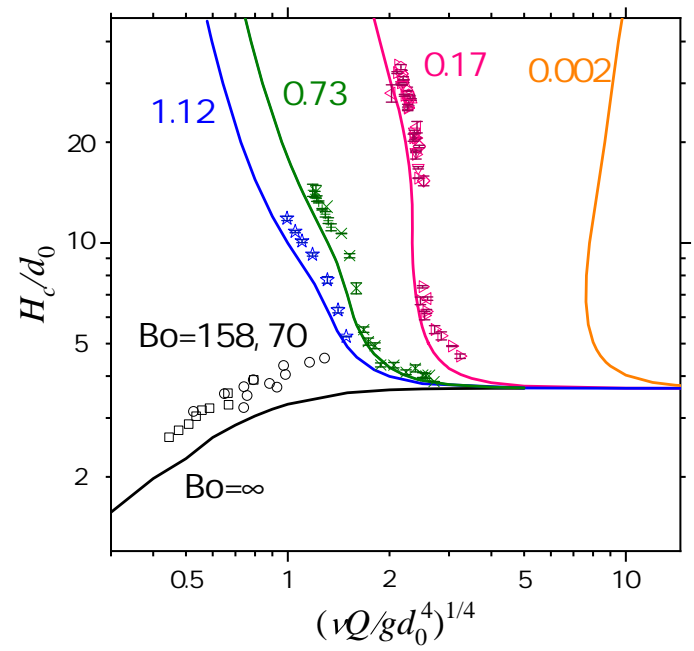

FIG. S3: Error bars added to fig. 3.

Note S4. Sources of experimental error. The errors bars for fig. 3 are shown in fig. S3. The sources of errors include (1) inaccuracy in determining the top and bottom boundary of the jet by eye during image processing; (2) fluctuations in pump flow rate; (3) hysteresis of the jet state due to viscosity; and (4) the difference between the nominal and real diameters of the needles used. To minimize the errors, (1) we have done multiple measurements; (2) we disturb the jet physically each time we change the dispensing height to allow a new steady rotating state to form; and (3) we increase the frequency of the measurements and decrease the increment of dispensing height for heights near the critical value $H_{c}$.

Note S5. Experimental observations for $B o \approx 0.032$. In fig. 4, the numerical curve for $\mathrm{Bo}=0.002$ suggests that there will be two onset heights. The nozzle diameter corresponding to $\mathrm{Bo}=0.002$ is $d_{0}=65 \mu \mathrm{m}$, which is 
too small for extruding viscous fluid at reasonable flow rates. Therefore we demonstrate the possibility of multiple onset heights using Bond number Bo $=0.032$ instead. By using a needle with diameter $d_{0}=0.26 \mathrm{~mm}$, we were able to observe two cessations of coiling during a single experiment with a fixed flow rate and a changing dispensing height, as shown in fig. S4 and the supplemental video. For one experiment with fixed flow rate of $4.5 \mathrm{~mL} / \mathrm{h}$, as the dispensing height decreases, an initially coiling jet moves from left to right on the dark yellow line in fig. S4. The first cessation of coiling occurs as the line enters below the concave-down part of the curve. As the height keeps decreasing and the jet enters the concave-up part of the curve, the jet starts to coil again. When the height decreases to very small values and goes beyond the near-vertical part of curve, the second cessation happens. In contrast to the existing literature where only slender jets buckle while wider jets do not, our new observations demonstrate the possibility of an unbuckled slenderer jet and a buckled wider jet due to the effect of surface tension. The video shows the state transition of the jet while decreasing the dispensing height with a fixed flow rate $4.5 \mathrm{~mL} / \mathrm{h}$ (The video only demonstrates the transition process and was not used to determine the critical height $H_{c}$, which was instead located by pausing at different heights and disturbing the jet to permit a new steady state to form).

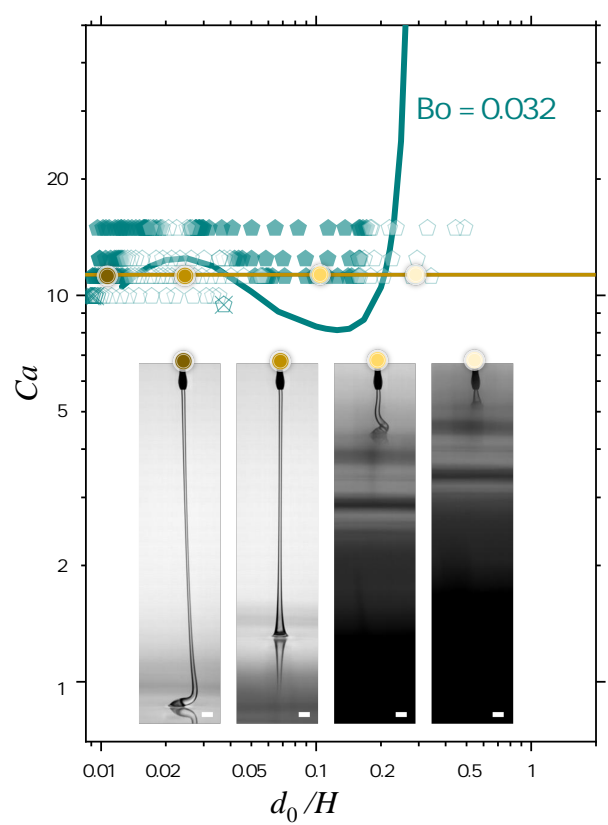

FIG. S4: Experimental results for $B o \approx 0.032$ with needle diameter $d_{0}=0.26 \mathrm{~mm}$ and viscosity $10 \mathrm{~Pa}$ s. Solid symbols represent coiling, open symbols correspond to stagnation flow, and crossed symbols correspond to jet breakup. The dark yellow line represents an experiment with $4.5 \mathrm{~mL} / \mathrm{h}$ flow rate and a varying dispensing height. The four dots on the line colored from dark yellow to light yellow represent four states of the jet as dispensing height decreases from left to right. The corresponding jet shapes are shown by the inset photographs below the same colored dots. Scale bars are $1 \mathrm{~mm}$.
Table S1. Parameter comparison with the literature.

\begin{tabular}{ccc}
\hline Reference & flow rate $(\mathrm{mL} / \mathrm{h})$ & nozzle diameter $(\mathrm{mm})$ \\
\hline$[2]$ & $7200-50400$ & $1.14-19.1$ \\
{$[4]$} & 5560 & 6 \\
{$[4]$} & $\leq 17640$ & 4 \\
{$[5]$} & $288-1080$ & $2-4$ \\
{$[1]$} & 291.6 & 6 \\
This study & $1.5-150$ & $0.26-1.55$ \\
\hline
\end{tabular}

Table S2. Jet slenderness just before coiling onset.

\begin{tabular}{cc}
\hline Referemce & $d / H$ just before onset of coiling \\
\hline$[3]$ & $3-4$ \\
{$[5]$} & $2-4$ \\
{$[6]$} & $5-6$ \\
This study (silicone oil) & up to $\approx 93$ \\
This study (Lecithin) & up to $\approx 35$ \\
\hline
\end{tabular}

* a). J. Tian and N. M. Ribe contributed equally to this work.

b). Corresponding author. ribe@fast.u-psud.fr

c). Corresponding author. ashum@hku.hk

[1] G. Barnes and R. Woodcock. Liquid rope-coil effect. Am. J. Physics, 26:205-209, 1958.

[2] J. O. Cruickshank. Viscous Fluid Buckling: A Theoretical and Experimental Analysis with Extensions to General Fluid Stability. PhD thesis, Iowa State University, 1980.

[3] J. O. Cruickshank and B. R. Munson. Viscous fluid buckling of plane and axisymmetric jets. J. Fluid Mech., 113:221-239, 1981.

[4] M. Habibi, S. H. Hosseini, M. H. Khatami, and N. M. Ribe. Liquid supercoiling. Phys. Fluids, 26:024101, 2014.

[5] M. Habibi, Y. Rahmani, D. Bonn, and N. M. Ribe. Buckling of liquid columns. Phys. Rev. Lett., 104:074301, 2010.

[6] M. Maleki, M. Habibi, R. Golestanian, N. M. Ribe, and D. Bonn. Liquid rope coiling on a solid surface. Phys. Rev. Lett., 93:214502, 2004.

[7] M. Le Merrer, D. Quéré, and C. Clanet. Buckling of viscous filaments of a fluid under compression stress. Phys. Rev. Lett., 109:064502, 2012.

[8] N. M. Ribe, M. Habibi, and D. Bonn. Stability of liquid rope coiling. Phys. Fluids, 18:084102, 2006b. 